\title{
Unrecognised postoperative residual curarisation in developing countries remains a common problem
}

\author{
S Chetty, ${ }^{1,2}$ MB ChB, FCA (SA), Cert Crit Care (SA), PhD; S Hassim, ${ }^{3}$ FCA (SA); H Perrie, ${ }^{2}$ MSc; J Scribante, ${ }^{2}$ PhD; M Parker, ${ }^{4}$ PhD \\ ${ }^{1}$ Department of Anaesthesiology and Critical Care, Faculty of Medicine and Health Sciences, Stellenbosch University, Cape Town, South Africa \\ ${ }^{2}$ Department of Anaesthesiology, Faculty of Health Sciences, University of the Witwatersrand, Johannesburg, South Africa \\ ${ }^{3}$ Private practice, Middelburg, Mpumalanga, South Africa \\ ${ }^{4}$ Department of Psychiatry, Faculty of Medicine and Health Sciences, Stellenbosch University, Cape Town, South Africa
}

Corresponding author: S Chetty (seanchetty@sun.ac.za)

\begin{abstract}
Background. Postoperative residual curarisation (PORC) is a potentially life-threatening complication of non-depolarising muscle relaxant use in anaesthesia. Quantitative neuromuscular monitoring has the potential to reduce the risk of PORC, but many anaesthetists rely on clinical assessment of neuromuscular function alone.

Objectives. To investigate the occurrence of PORC in the recovery room at an academic hospital in Gauteng, South Africa. Additionally, this prospective, cross-sectional study aimed to determine the extent of intraoperative neuromuscular monitoring and to assess clinical factors that may influence the risk of PORC.

Methods. Patient characteristics and clinical information, including whether neuromuscular monitoring had been conducted, were recorded. Patients were evaluated by clinical assessment and determination of a quantitative train-of-four ratio (TOFR). PORC assessment methods were compared and clinical characteristics were analysed for association with TOFR status.

Results. The incidence of PORC (TOFR $<0.9$ ) in the study group was $45.5 \%$; however, $78 \%$ passed the clinical assessment. The specificity of clinical assessment to detect residual paralysis was $24 \%$. Notably, only 3 patients $(6 \%)$ were monitored intraoperatively for neuromuscular function. Advanced age $(p=0.011, r=-0.34)$ and female gender $(p=0.001)$ were shown to be associated with a TOFR $<0.7$.

Conclusions. PORC is more common in practice than currently recognised. This deduction is supported by the low rate of intraoperative neuromuscular monitoring conducted and the high pass rate on clinical assessment. Routine use of quantitative monitoring of neuromuscular function should be encouraged to minimise the risk of this serious yet preventable condition.
\end{abstract}

S Afr Med J 2020;110(11):1134-1138. https://doi.org/10.7196/SAMJ.2020.v110i11.14759

Non-depolarising muscle relaxants (NDMRs) have been used in anaesthesia for nearly 80 years. Incomplete reversal leading to postoperative residual curarisation (PORC) may result in potentially life-threatening complications following surgery. ${ }^{[1]}$ The use of longacting NDMRs is known to increase the likelihood of PORC, , $2-4]^{-}$ but intermediate-acting NDMR use has also been associated with substantial risk of residual paralysis. ${ }^{[5-7]}$ Residual effects of neuromuscular blocking agents delay discharge of patients from the operating theatre recovery room and place them at risk of developing a number of clinical complications postoperatively. Clinical consequences of PORC include an increased risk of respiratory complications, prolonged intubation times and increased patient morbidity. ${ }^{[8]}$ General factors that increase the risk of PORC include the use of high doses of NDMRs, hypothermia, and premature or delayed administration of anticholinesterase reversal agents. ${ }^{[6-7]}$ NDMRs should therefore be used with caution in patients who are elderly ${ }^{[9]}$ or obese, ${ }^{[10]}$ have sleep apnoea $^{[11]}$ and who have neuromuscular or respiratory pathology, ${ }^{[12]}$ owing to the risks associated with these drugs.

Peri- and postoperative neuromuscular monitoring has the potential to minimise the risk of PORC and improve patient outcomes. ${ }^{[8,13,14]}$ In 2018, a consensus statement was released recommending the quantitative monitoring of neuromuscular function whenever a neuromuscular blocker is administered. ${ }^{[15]}$

Evaluation of adequate neuromuscular recovery is routinely performed in the operating room using peripheral nerve stimulation and patient assessment. ${ }^{[16,17]}$ Common clinical parameters used to assess adequate motor function include a $\geq 5$-second hand grip, the ability to cough, a head lift for $\geq 5$ seconds, an acceptable minute ventilation, and negative inspiratory pressures $>-30 \mathrm{~cm} \mathrm{H}_{2} \mathrm{O} \cdot{ }^{[3,18]}$ Qualitative monitoring and clinical assessment, however, have been shown to be unreliable ${ }^{[8]}$ and unable to establish readiness for tracheal extubation. ${ }^{[15]}$ In addition, the patient should be awake and co-operative, and no residual anaesthetic drug should be present for accurate assessment of these parameters. Such optimal conditions are rarely accomplished in routine practice. Other factors, such as pain during the postoperative period, complicate assessment even for patients who are awake.

Quantitative measurement of the train-of-four ratio (TOFR) ${ }^{[19]}$ using techniques such as acceleromyography or electromyography is considered highly accurate and is required for the detection of minimal block, defined as a TOFR $\geq 0.9 .^{[8,15]}$ Residual paralysis, identified by a TOFR $<0.9$, is associated with complications such as hypoxia, weakness, impaired swallowing, and respiratory failure that can lead to brain death. However, few medical facilities have the necessary evaluation equipment readily available, and clinical assessment alone is often considered sufficient by many anaesthetists worldwide. ${ }^{[18,20,21]}$ Similarly, South African (SA) anaesthetists generally rely on clinical judgement of adequate motor function, and this is likely to result in underestimation of the occurrence of PORC in clinical practice. 


\section{Objectives}

To investigate the occurrence of PORC in the recovery room for patients who received intermediate-acting NDMRs intraoperatively at Chris Hani Baragwanath Academic Hospital (CHBAH), an academic training hospital in Johannesburg, SA. Moreover, we aimed to identify clinical parameters that may be associated with residual paralysis in a resource-limited setting.

\section{Methods}

This prospective study was conducted at $\mathrm{CHBAH}$, an academic hospital that serves as a primary referral centre for the greater Gauteng area. The study sample consisted of 55 American Society of Anesthesiologists 1 - 3 patients who had undergone elective surgery, received an intermediate-acting NDMR, and were extubated postoperatively. The study sample size was calculated based on $90 \%$ power and an estimated PORC incidence of $20 \%$. Ethics approval for the research was granted by the Human Research Ethics Committee of the University of the Witwatersrand (ref. no. M140360). Research participants were recruited prior to surgery and written informed consent was obtained. Patients were excluded from the study if they were $<18$ years of age or were unable to speak English, or if there was no access to either ulnar nerve.

Data were collected between December 2014 and February 2015. Participants were seen in the operating theatre recovery room after patient assessment had been completed by the attending anaesthetist. Patient characteristics and clinical information were recorded, including the intraoperative NDMR used and whether neuromuscular monitoring had been performed during the surgery. Clinical assessment of neuromuscular function included the ability to sustain a head lift for $\geq 5$ seconds, hand-grip strength ( $\geq 5$-second hold), the ability to cough, and pulse oximetry. Participants who could maintain the head lift and hand grip, who were able to cough and who had an oxygen saturation of at least $90 \%$ passed the clinical assessment.

Potential PORC was determined independently by the researcher using a TOFWatch SX (Organon Laboratories, UK) accelerometer. Electrodes were placed over the ulnar nerve on the volar aspect of the wrist and the acceleration sensor was connected to an unrestricted thumb. The negative electrode was placed $1 \mathrm{~cm}$ proximal to the point at which the proximal flexion crease of the wrist crosses the flexor carpi ulnaris tendon. The positive electrode was placed $2-3 \mathrm{~cm}$ proximal to the negative electrode. The TOFR was generated on the accelerometer by application of a submaximal stimulus of $30 \mathrm{~mA}$. As only a fraction of fibres were induced in a given nerve bundle, the twitch height was reduced, but the ratio measured between the twitches remained the same. Three consecutive measurements taken 20 seconds apart were recorded, and the average was calculated for the final TOFR.

Excel 2010 (Microsoft, USA) was used for initial data capture, and subsequent statistical analyses were completed using SPSS Statistics version 25.0 (IBM, USA). Chi-square analyses were performed to explore potential associations between the clinical and quantitative variables. Where necessary, the Freeman-Halton extension of Fisher's exact test was applied. Continuous variables including age and oxygen saturation

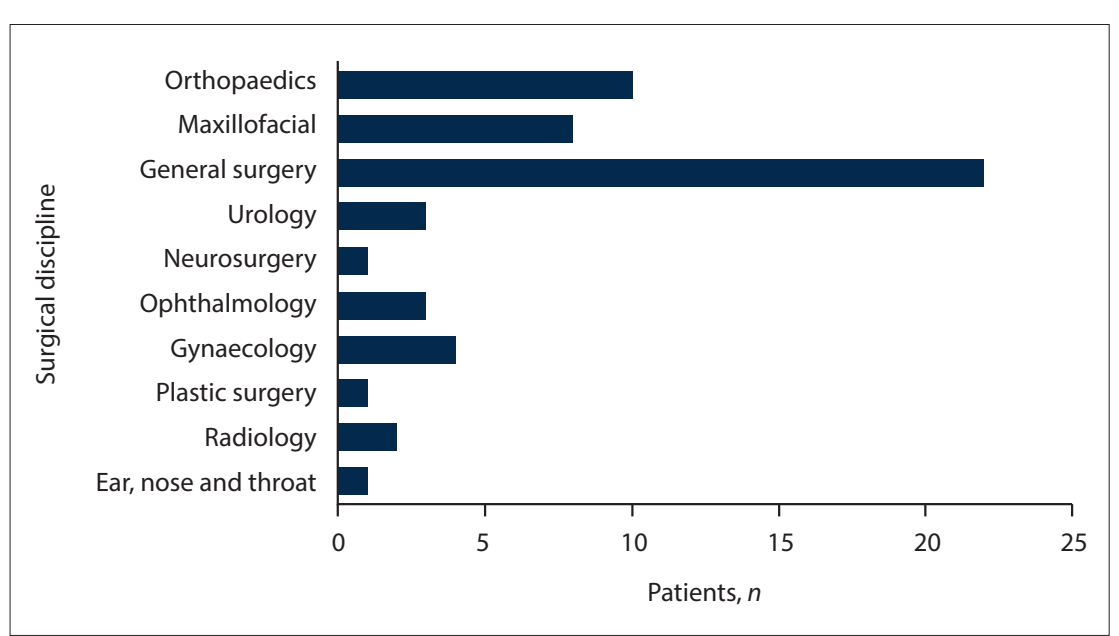

Fig. 1. Range of surgical disciplines.

\begin{tabular}{ll} 
Table 1. Patient characteristics & \\
\hline Characteristic & \\
\hline Age (years) & $20-79 ; 44(15.8)$ \\
$\quad$ Range; mean (SD) & $45(81.8)$ \\
$\quad<60, n(\%)$ & $10(18.2)$ \\
$\quad \geq 60, n(\%)$ & \\
Gender, $n(\%)$ & $30(54.5)$ \\
$\quad$ Male & $25(45.5)$ \\
Female & \\
NDMR type, $n$ (\%) & $6(10.9)$ \\
$\quad$ Atracurium & $9(16.4)$ \\
Cisatracurium & $32(58.2)$ \\
Rocuronium & $8(14.5)$ \\
Vecuronium & \\
Intraoperative neuromuscular monitoring, $n(\%)$ & $3(5.5)$ \\
Yes & $52(94.5)$ \\
No & \\
Reversal agent administered (neostigmine), $n(\%)$ & $48(87.3)$ \\
Yes & $7(12.7)$ \\
No & \\
SD = standard deviation; NDMR = non-depolarising muscle relaxant. & \\
&
\end{tabular}

were analysed using Student's $t$-test and the Mann Whitney $U$-test. The two methods used to assess PORC in these participants, based on either clinical assessment criteria or the TOFR, were compared using McNemar paired $\chi^{2}$ analysis. In addition, the strength of association (Eta squared) was calculated. Sensitivity and specificity analyses for the clinical assessment were also performed. All comparisons were two-sided, and a $p$-value of $<0.05$ was considered statistically significant.

\section{Results}

The study participants were from a range of different surgical disciplines, with $40 \%$ undergoing general surgery (Fig. 1).

A total of 55 patients were included in the study. Participant characteristics and clinical information are summarised in Table 1. 
Participant responses on postoperative assessment are shown in Table 2. The study participant temperatures ranged from $34.1^{\circ} \mathrm{C}$ to $38.4^{\circ} \mathrm{C}$, and $4(7.3 \%)$ of the participants were found to be hypothermic. Supplemental oxygen $(8 \mathrm{~L} / \mathrm{min})$ was applied to 42 participants $(76.4 \%)$ in the recovery room. Mean (standard deviation (SD)) oxygen saturation was $96.4 \%$ (2.9) and ranged from $85 \%$ to $100 \%$. A single participant had an oxygen saturation of $<90 \%$. On clinical assessment only 4 participants (7.3\%) were unable to cough, and 9 (16.4\%) and 11 $(20.0 \%)$ were unable to sustain a hand grip or head lift, respectively, for $\geq 5$ seconds.

Intraoperative neuromuscular monitoring was recorded on the anaesthetic chart for 3 participants (5.4\%). In total, 12 participants $(21.8 \%)$ failed the study clinical assessment, thereby meeting the clinical criteria for PORC. In contrast, 25 participants $(45.5 \%)$ met the criteria for PORC on TOF monitoring, i.e. a TOFR $<0.9$. Average TOFRs ranged from 0.3 to 1 with a mean (SD) of $0.85(0.15)$. Fig. 2 shows the number of participants in each of four TOFR categories, namely $<0.5,0.5$ $0.69,0.7-0.89$ and $\geq 0.9$.

\section{Clinical associations with TOFR threshold value}

Potential associations between clinical parameters and average TOFR were investigated. Participants were initially grouped based on a TOFR threshold value of 0.9 indicative of the presence or absence of PORC. However, no significant association between the clinical parameters assessed and TOFR status could be demonstrated in this cohort.

Potential associations were further interrogated using TOFR threshold values of 0.8 and 0.7 , respectively. Similar results were obtained for the majority of variables assessed; however, significant associations were observed for age (TOFR $<0.7 ; p=0.009$, $r=-0.34$ ) and gender (TOFR $<0.7 ; p=0.001$, Fisher's exact test and TOFR $<0.8 ; \chi^{2}=13.22$, $\mathrm{df}=1, p=<0.001)$. Lower TOFRs were associated with older age and female gender.

\section{Comparison of PORC assessment methods}

As mentioned previously, a higher percentage of participants met the criteria for PORC using the TOFR assessment method than the clinical assessment method (45.5\% v. $22 \% ; p=0.015$, McNemar test, exact $p$-value). On comparison of participants grouped by TOFR status $(<0.9$ and $\geq 0.9)$, participant pass rate on clinical assessment was not significantly different. Similarly, average TOFR status did not differ between

\begin{tabular}{|c|c|c|}
\hline Postoperative assessment & $n(\%)$ & Mean (range; SD) \\
\hline Temperature $\left({ }^{\circ} \mathrm{C}\right)$ & $55(100)$ & $36.3(34.1-38.4 ; 0.9)$ \\
\hline$<35$ & $4(7.3)$ & \\
\hline$\geq 35$ & $51(92.7)$ & \\
\hline Oxygen saturation (\%) & $55(100)$ & $96.4(85-100 ; 2.9)$ \\
\hline \multicolumn{3}{|l|}{ Oxygen applied (8 L/min) } \\
\hline No & $13(23.6)$ & \\
\hline Yes & $42(76.4)$ & \\
\hline Head-lift time (s) & $55(100)$ & $4.3(0-5 ; 1.5)$ \\
\hline$<5$ & $11(20.0)$ & \\
\hline$\geq 5$ & $44(80.0)$ & \\
\hline Hand-grip time (s) & $55(100)$ & $3.9(0-5 ; 1.6)$ \\
\hline$<5$ & $9(16.4)$ & \\
\hline$\geq 5$ & $46(83.6)$ & \\
\hline \multicolumn{3}{|l|}{ Ability to cough } \\
\hline Yes & $51(92.7)$ & \\
\hline No & $4(7.3)$ & \\
\hline \multicolumn{3}{|l|}{ Clinical assessment } \\
\hline Pass & $43(78.2)$ & \\
\hline Fail & $12(21.8)$ & \\
\hline Average TOFR & $55(100)$ & $0.85(0.3-1 ; 0.15)$ \\
\hline$<0.9$ & $25(45.5)$ & $0.67(0.3-0.89 ; 0.17)$ \\
\hline$\geq 0.9$ & $30(54.5)$ & $0.94(0.9-1 ; 0.03)$ \\
\hline
\end{tabular}

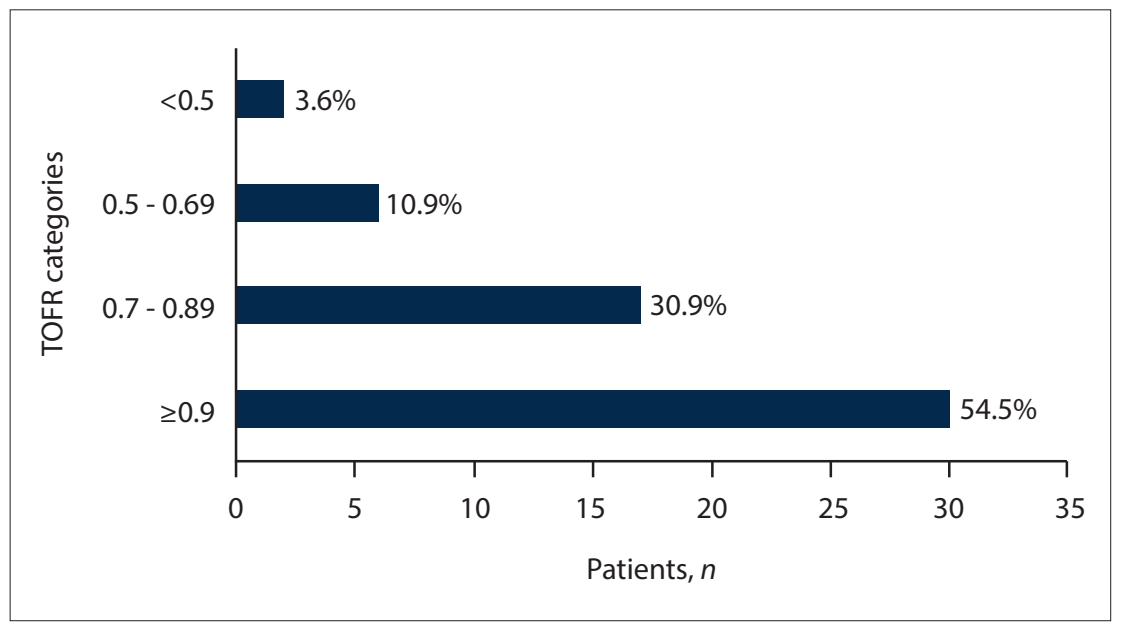

Fig. 2. Proportion of patients in each TOFR category. (TOFR = train-of-four ratio. $)$

participants grouped by clinical assessment; however, TOFR status was moderately associated with clinical assessment outcome $\left(\operatorname{Eta}(\eta)=0.7 ; \eta^{2}=0.49\right)$.

\section{Clinical assessment sensitivity and specificity analyses}

Applying the TOFR assessment as the gold standard, ${ }^{[8]} 25$ of the 55 participants (45.5\%) were incorrectly assigned a pass or fail status on clinical assessment by the attending anaesthetist at the end of surgery. The sensitivity and specificity of the clinical assessment were determined to be $80 \%$ and $24 \%$, respectively.

\section{Discussion}

The occurrence of residual paralysis in the recovery room after elective surgery has not been investigated at $\mathrm{CHBAH}$ previously. International studies over the last 30 years have reported incidences ranging from $17 \%$ to $64 \%{ }^{[3,18,22]}$ During this time period, methods for measuring TOF fade have been improved, and the TOFR criteria for exclusion of clinically relevant PORC have been adapted. Early studies suggested that a TOFR of 0.7 correlated with clinically acceptable signs of recovery. ${ }^{[2,23]}$ However, the recommended threshold value has since been increased to 0.9 , as impaired 
respiratory function and muscle weakness have been associated with lower ratio values. ${ }^{[24]}$

In our cohort, the occurrence of PORC, defined as a TOFR $<0.9$, was 46\%. In 2004, a study from Universitas Hospital in Bloemfontein, SA, reported a $42.9 \%$ incidence of PORC using a TOFR $<0.9$ and $17 \%$ when lowering the TOFR to $<0.8 \cdot{ }^{[25]}$ Similarly, data from the current study indicate a $22 \%$ incidence of residual paralysis using a TOFR $<0.8$. Another SA study, which evaluated postoperative neuromuscular function in 70 participants from Inkosi Albert Luthuli Central Hospital, Durban, found that $26.8 \%$ of participants presented with a TOFR $<0.9 .{ }^{[26]}$ Our data support numerous other studies ${ }^{[4,5,27,28]}$ reporting a high incidence of residual paralysis in participants arriving in the post-anaesthesia care unit. Despite the evidence, the risk of PORC remains underestimated among anaesthetists in SA.

According to our data, only $6 \%$ of participants in our cohort were monitored for neuromuscular blockade. Such practices are likely to contribute to the lack of recognition of PORC. Internationally, neuromuscular monitoring for PORC is not commonly practised among anaesthetists. A survey from Italy that included 754 anaesthetists revealed that $73 \%$ relied on assessment of clinical parameters alone. ${ }^{[20]}$

In the current study, $78 \%$ of participants passed the study's clinical assessment meeting the criteria for adequate reversal of their neuromuscular blockade. However, 19 of the participants who passed had a TOFR $<0.9$. Therefore, $35 \%$ of participants were inaccurately identified as reversed by the attending physician. In addition, 6 participants who failed the study clinical assessment in the recovery room had a TOFR $\geq 0.9$. The sensitivity of the clinical assessment in this study was $80 \%$. Clinical assessment was able to identify the majority of those who were reversed. However, the specificity of the clinical assessment was low (24\%). A large proportion of the participants who did not meet the criteria for reversal on TOF monitoring were clinically assessed to be reversed (76\%). The insufficiencies associated with clinical assessment have been carefully reviewed, ${ }^{[3,8]}$ and in general, these tests have been shown to be unreliable. ${ }^{[10,27]}$ Our results confirm that clinical assessment alone failed to identify the majority of participants with residual paralysis. Of these, $4(21 \%)$ had a TOFR $<0.7$. Although the side-effects related to a TOFR $>0.7$ may not be life-threatening, residual muscle weakness may have important clinical consequences, including a higher risk of atelectasis, pneumonia and microaspirations. ${ }^{[1]}$ A further $20 \%$ of participants who had a TOFR $>0.9$ were diagnosed with PORC on clinical assessment. Ultimately, for both groups of participants, delays in discharge from the recovery room, ${ }^{[29]}$ prolonged intubation times $^{[30]}$ and poor patient satisfaction are inevitable consequences of this misdiagnosis.

In the current study, the clinical parameters assessed were not shown to be associated with TOFR status. However, older age and female gender were associated with a TOFR $<0.7$. These results concur with previously published international data. It has been demonstrated previously that older patients ( $\geq 65$ years) were twice as likely as younger patients to experience residual neuromuscular paralysis following NDMR administration. ${ }^{[31]}$ In a different study, Kaan et al. ${ }^{[32]}$ demonstrated that the risk of postoperative residual neuromuscular block was increased in women (odds ratio 7.25) in their study population.

No significant association was observed between TOFR and oxygen saturation level. Reduced oxygen saturation levels, however, may occur for medical reasons unrelated to NMDR administration. The average oxygen saturation among the participants in our study was $96 \%$. Supplemental oxygen was required by $24 \%$ of participants during recovery, and all participants with a TOFR $<0.7$ required supplemental oxygen. One patient, who had a TOFR of only 0.3 , maintained an oxygen saturation of $85 \%$. This individual passed the other criteria for clinical assessment. In general, participants with a TOFR $<0.6$ should be able to lift their head and maintain a hand grip for 3 seconds, stick out their tongues and open their eyes wide, but may display reduced vital capacity and negative inspiratory pressure. ${ }^{[19,23]}$

The development of PORC was not found to be associated with the choice of NDMR. However, of the participants who received cisatracurium, a slightly higher percentage $(56 \%)$ met the criteria for PORC. In addition, cisatracurium had been administered to the patient who presented in the recovery room with a TOFR of only 0.3 . A previous study by Maybauer et al. ${ }^{[33]}$ demonstrated that cisatracurium was associated with a higher incidence of residual paralysis than rocuronium (56\% v. $44 \%)$. Cisatracurium also has a longer duration of effect than atracurium, ${ }^{[34]}$ and only $33 \%$ of participants who received atracurium presented with PORC.

The administration of an anticholinesterase to antagonise the effects of the neuromuscular blocking agent has previously been found to be necessary to reduce the incidence of PORC..$^{[7,28]}$ In our study, although the use of a reversal agent was not significantly associated with TOFR status, it is worth noting that, of the 7 participants who did not receive neostigmine, 6 had a TOFR $\leq 0.9$. As the number of participants who did not receive a reversal agent is small, it is possible that for a larger sample size, application of an anticholinesterase would show significant association with TOFR status. Although not investigated in this study, the use of sugammadex has the potential to reduce the incidence of PORC. ${ }^{[35]}$

\section{Conclusions}

The prevalence of PORC in our study is higher than currently recognised by clinical assessment. Relying on subjective assessment of neuromuscular function alone results in a large proportion of patients presenting to the recovery room with a TOFR $<0.9$. The use of quantitative neuromuscular monitoring should be encouraged both peri- and postoperatively, and awareness regarding residual paralysis in the recovery room should be improved. The South African Society of Anaesthesiologists Practice Guidelines, 2018 revision, ${ }^{[36]}$ recommend a peripheral nerve stimulator as an essential piece of equipment in surgery to monitor neuromuscular function. Such monitoring forms part of the minimum recommended requirements for safe practice. Given that PORC can lead to major morbidity, yet is a preventable condition, it is essential to implement the best strategies to recognise and manage it. The investigation described is limited in that this was a single-centre study; however, the results are generalisable worldwide, as the literature confirms that the incidence of intraoperative monitoring is low in institutions across the globe. ${ }^{[20]}$ Future studies examining larger cohorts of participants from multiple centres would also be valuable to examine potential clinical parameters that predispose to a risk of PORC in low- and middle-income countries.

Declaration. The research for this study was done in partial fulfilment of the requirements for SH's MMed (Anaes) degree at the University of the Witwatersrand.

Acknowledgements. We thank Dr Des Lines for the loan of the TOF-Watch SX used for TOF measurements in the recovery room.

Author contributions. Protocol development: SC, SH, HP, JS; data collection: $\mathrm{SH}$; data analysis: $\mathrm{SH}$ and MP; interpretation of results: $\mathrm{SC}, \mathrm{SH}$ and MP; manuscript preparation: $\mathrm{SC}, \mathrm{SH}$ and MP; manuscript review: $\mathrm{SC}$, SH, HP, JS, MP. 
Funding. This work was supported by funding provided by the Department of Anaesthesiology, University of the Witwatersrand.

Conflicts of interest. SC served on the speaker bureau for the following organisations that have commercial interests related to neuromuscular blocking agents: Adcock Ingram Critical Care, MSD South Africa, Fresenius Kabi South Africa, Aspen Pharmaceuticals.

1. Murphy GS, Brull SJ. Residual neuromuscular block: Lessons unlearned. Part I: Definitions, incidence, and adverse physiologic effects of residual neuromuscular block. Anesth Analg 2010;111(1):120-128. and adverse physiologic effects of residual neuro
https://doi.org/10.1213/ANE.0b013e3181da832d

2. Viby-Mogensen MDJ, Chraemmer Jørgensen MDB, Ørding MDH. Residual curarization in the recovery room. Anesthesiol J Am Soc Anesthesiol 1979;50(6):539-541. https://doi. in the recovery room. Anesthesiol J
org/10.1097/00000542-197906000-00014

3. Murphy GS. Residual neuromuscular blockade: Incidence, assessment, and relevance in the postoperative period. Minerva Anestesiol 2006;72(3):97-109.

4. Naguib M, Kopman AF, Ensor JE. Neuromuscular monitoring and postoperative residual curarisation: A meta-analysis. Br J Anaesth 2007;98(3):302-316. https://doi.org/10.1093/bja/ael386

5. Hayes AH, Mirakhur RK, Breslin DS, Reid JE, McCourt KC. Postoperative residual block after intermediate-acting neuromuscular blocking drugs. Anaesthesia 2001;56(4):312-318. https://doi. org/10.1046/j.1365-2044.2001.01921.x

6. Brull SJ, Murphy GS. Residual neuromuscular block: Lessons unlearned. Part II: Methods to reduce the risk of residual weakness. Anesth Analg 2010;111(1):129-140. https://doi.org/10.1213/ ANE.0b013e3181da8312

7. Xie M, Wang D-X, Geng Z-Y. Prevalence and risk factors of postoperative residual curarization in patients arriving at postanesthesia care unit after general anesthesia: A prospective cohort study. patients arriving at postanesthesia care unit after general anesthesia: A prospec
J Anesth Perioper Med 2014;1(2):72-78. https://doi.org/10.24015/JAPM.2014.0012

8. Plaud B, Debaene B, Donati F, Marty J. Residual paralysis after emergence from anesthesi Anesthesiology 2010;112(4):1013-1022. https://doi.org/10.1097/ALN.0b013e3181cded07

9. Murphy GS, Szokol JW, Avram MJ, et al. Residual neuromuscular block in the elderly: Incidence and clinical implications. Anesthesiol J Am Soc Anesthesiol 2015;123(6):1322-1336. https://doi. org/10.1097/ALN.000000000000086

10. Saager L, Maiese EM, Bash LD, et al. Incidence, risk factors, and consequences of residual neuromuscular block in the United States: The prospective, observational, multicenter RECITE-US study. J Clin Anesth 2019;55:33-41. https://doi.org/10.1016/j.j.jlinane.2018.12.042

11. Hafeez KR, Tuteja A, Singh M, et al. Postoperative complications with neuromuscular blocking drugs and/or reversal agents in obstructive sleep apnea patients: A systematic review. BMC Anesthesiol 2018; 18(1):1-11. https://doi.org/10.1186/s12871-018-0549-x

12. Schieren M, Defosse J, Böhmer A, Wappler F, Gerbershagen MU. Anaesthetic management of patients with myopathies. Eur J Anaesthesiol 2017;34(10):641-649. https://doi.org/10.1097/ EJA.0000000000000672

13. Fruergaard K, Viby-Mogensen J, Berg H, El-Mahdy AM. Tactile evaluation of the response to double burst stimulation decreases, but does not eliminate, the problem of postoperative residual paralysis. burst stimulation decreases, but does not eliminate, the problem of postoperative residual paralysis,
Acta Anaesthesiol Scand 1998:42(10):1168-1174. https://doi.org/10.1111/j.1399-6576.1998.tb05271.x Acta Anaesthesiol Scand 1998;42(10):1168-1174. https:// doi.org/10.1111/j.1399-6576.1998.tb05271.x
4. Gätke MR, Viby-Mogensen J, Rosenstock C, Jensen FS, Skovgaard LT. Postoperative muscle paralysis after rocuronium: Less residual block when acceleromyography is used. Acta Anaesthesiol Scand 2002;46(2):207-213. https://doi.org/10.1034/j.1399-6576.2002.460216.x

15. Naguib M, Brull SJ, Kopman AF, et al. Consensus statement on perioperative use of neuromuscular monitoring. Anesth Analg 2018;127(1):71-80. https://doi.org/10.1213/ANE.0000000000002670

16. McGrath CD, Hunter JM. Monitoring of neuromuscular block. Update Anaesth 2006;25(1):42-46. https://doi.org/10.1093/bjaceaccp/mki067

17. Viby-Mogensen J. I: Postoperative residual curarization and evidence-based anaesthesia. $\mathrm{Br} J$ Anaesth 2000;84(3):301-303. https://doi.org/10.1093/oxfordjournals.bja.a013428

18. Grayling M, Sweeney BP. Recovery from neuromuscular blockade: A survey of practice. Anaesthesia 2007;62(8):806-809. https://doi.org/10.1111/1.1365-2044.2007.05101.x
19. Ghai B, Makkar JK, Wig J. Neuromuscular monitoring: A review. J Anaesthesiol Clin Pharmacol 2006;22(4):347-356

20. Di Marco P, della Rocca G, Iannuccelli F, Pompei L, Reale C, Pietropaoli P. Knowledge of residual curarization: An Italian survey. Acta Anaesthesiol Scand 2010;54(3):307-312. https://doi.org/10.1111/ j.1399-6576.2009.02131.x

21. Philips S, Stewart PA, Bilgin AB. A survey of the management of neuromuscular blockad monitoring in Australia and New Zealand. Anaesth Intensive Care 2013;41(3):374-379. https://doi. org/10.1177/0310057X1304100316

22. Kopman AF, Eikermann M. Antagonism of non-depolarising neuromuscular block: Current practice. Anaesthesia 2009;64(s1):22-30. https://doi.org/10.1111/j.1365-2044.2008.05867

23. Ali HH, Wilson RS, Savarese JJ, Kitz RJ. The effect of tubocurarine on indirectly elicited train-of-four muscle response and respiratory measurements in humans. Br J Anaesth 1975;47(5):570-574. https:// doi.org/10.1093/bja/47.5.570

24. Kopman AF, Ng J, Zank LM, Neuman GG, Yee PS. Residual postoperative paralysis: Pancuronium versus mivacurium, does it matter? Anesthesiol J Am Soc Anesthesiol 1996;85(6):1253-1259. https:// doi.org/10.1097/00000542-199612000-0000

25. Nell W, Stevenson N, Ridgard T, van der Westhuizen F, Diedericks J, Joubert G. Post-operative neuromuscular function of patients receiving non-depolarising muscle relaxants at Universitas Hospital, Bloemfontein, South Africa. South Afr J Anaesth Analg 2004;10(1):6-8. https://doi.org/10. 1080/22201173.2004.10872344

26. Invernizzi JRR, Gopalan PD. Postoperative neuromuscular function following non-depolarising muscle blockade in patients at Inkosi Albert Luthuli Central Hospital, Durban. South Afr J Anaesth Analg 2016;22(4):121-124. https://doi.org/10.1080/22201181.2016.1201293

27. Debaene B, Plaud B, Dilly MP, Donati F. Residual paralysis in the PACU after a single intubatin dose of nondepolarizing muscle relaxant with an intermediate duration of action. Anesthesiology 2003;98(5):1042-1048. https://doi.org/10.1097/00000542-200305000-00004

28. Baillard C, Gehan G, Reboul-Marty J, Larmignat P, Samama CM, Cupa M. Residual curarizatio in the recovery room after vecuronium. Br J Anaesth 2000;84(3):394-395. https://doi.org/10.1093 oxfordjournals.bja.a013445

29. Butterly A, Bittner EA, George E, Sandberg WS, Eikermann M, Schmidt U. Postoperative residual curarization from intermediate-acting neuromuscular blocking agents delays recovery room discharge. Br J Anaesth 2010;105(3):304-309. https://doi.org/10.1093/bja/aeq157

30. Thomas R, Smith D, Strike P. Prospective randomised double-blind comparative study of rocuronium and pancuronium in adult patients scheduled for elective 'fast-track' cardiac surgery involving hypothermic cardiopulmonary bypass. Anaesthesia 2003:58(3):265-271. https://doi.org/10.1046/ j.1365-2044.2003.30362.x

31. Pietraszewski P, Gaszyński T. Residual neuromuscular block in elderly patients after surgical procedures under general anaesthesia with rocuronium. Anaesthesiol Intensive Ther 2013;45(2):77 81. https://doi.org/10.5603/AIT.2013.0017

32. Kaan N, Kocaturk O, Kurt I, Cicek H. The incidence of residual neuromuscular blockade associated with single dose of intermediate-acting neuromuscular blocking drugs. Middle East J Anesthesiol 2012;21(4):535-542.

33. Maybauer DM, Geldner G, Blobner M, et al. Incidence and duration of residual paralysis at the end of surgery after multiple administrations of cisatracurium and rocuronium. Anaesthesia 2007;62(1):1217. https://oi.org/10.1111/j.1365-2044.2006.04862.x

34. Atef H, Helmy A, El-Nasr Ma, El-Kasaby A. Cisatracurium in different doses versus atracuriun during general anesthesia for abdominal surgery. Saudi J Anaesth 2010;4(3):152. https://doi. org/10.4103/1658-354X.71571

35. Abrishami A, Ho J, Wong J, Yin L, Chung F. Sugammadex, a selective reversal medication fo preventing postoperative residual neuromuscular blockade. Cochrane Database Syst Rev 2009, Issue 4. Art. No.: CD007362. https://doi.org/10.1002/14651858.CD007362.pub2

36. South African Society for Anaesthesiologists. SASA Practice Guidelines 2018 revision. South Afr Anaesth Analg 2018;24(2):S1-S119.

Accepted 21 May 2020 